\title{
Ausdruck eines sich erschöpfenden Selbst
}

\section{Markus Binswanger}

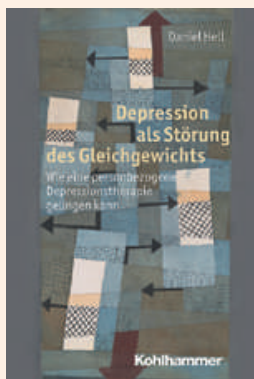

Daniel Hell

Depression als Störung des Gleichgewichts

Wie eine personbezogene Depressionstherapie gelingen kann

Stuttgart: Kohlhammer

Verlag; 2012

176 Seiten, 40.90 CHF

ISBN 978-3-17-021713-3

Korrespondenz:

Dr. med. Markus Binswanger Obere Bahnhofstrasse 9

CH-9500 Wil

markus.binswanger[at]clienia.ch
In der wachsenden Flut von Publikationen der letzten Jahre zu affektiven Erkrankungen ragt Daniel Hells «Welchen Sinn macht Depression?» heraus. Das 1994 veröffentlichte, inzwischen in 15. Auflage herausgegebene und in viele Sprachen übersetzte Buch ist zu einem Standardwerk geworden. Der Autor erschliesst darin das depressive Geschehen in vielfältigen Facetten als grundlegende menschliche Reaktionsmöglichkeit und - dieser Zugang ist neu und ungewohnt - als verborgene Botschaft, die es in einem integrativen Therapieansatz zu entschlüsseln gilt. Nun legt der Autor eine weitere, demnächst in zweiter Auflage erscheinende Publikation vor, die «Depression als Störung des Gleichgewichts» konzeptualisiert. Dem Leser, der Leserin stellt sich somit die Frage: Werden hier substantiell neue diagnostische und therapeutische Depressionsaspekte vorgetragen? Und weiter: Lohnt es sich überhaupt, angesichts der heute unüberblickbaren Zahl depressionstherapeutischer Konzepte, das Thema in einem neuen Buch weiterzuschreiben?

Die von Daniel Hell gewählte Zielsetzung ist anspruchsvoll: «Ich suche in diesem Buch die empirischen Erkenntnisse der Depressionsforschung der letzten Jahre und Jahrzehnte in ein Gesamtkonzept einzuordnen, das die Person ins Zentrum stellt. Ich verstehe Depression als das Ringen einer Person um ihr Gleichgewicht».

Das Buch gliedert sich in 11 Kapitel, die jedes für sich gelesen werden kann. Es beginnt mit grundsätzlichen Fragen und mündet ein in eine störungsbezogene depressionstherapeutische Praxeologie. Im ersten Kapitel stellt der Autor ein personenbezogenes Depressionsverständnis dar, das dem subjektiven Erlebnisraum - der sog. Perspektive der ersten Person - zentrale Beachtung schenkt. Dieser individuelle Ansatz wird einer evidenzbasierten Depressionsbehandlung gegenübergestellt und am Beispiel ausgewählter Depressionsformen erläutert. Ein pragmatisches Depressionsmodell mit wertvollen schematischen Darstellungen hilft dem Leser, sich in den komplexen biologischen und psychosozialen Krankheitsdimensionen zu orientieren und darauf aufbauend eine integrative Gesamtschau einzunehmen. Dem gleichen Zweck dienen sorgfältig ausgewählte und didaktisch geschickt dargestellte klinische Fallbeispiele. Schwerpunkte des zweiten, behandlungspraktischen Teils sind: Bindungsverhalten und therapeutische Beziehungsgestaltung, der Umgang mit Scham und Beschämung und das psychotherapeutische Arbeiten am Selbstbild. Der Leser wird hier mit wichtigen depressionsgeschichtlichen Aspekten und auch mit philosophischen Fragestellungen zum «Selbst» konfrontiert, die sich als durchaus praxisrelevant erweisen. Bekannte und bewährte, vom Autor bereits früher dargestellte depressionsspezifische Therapieansätze erfahren wertvolle ergänzende Hinweise auf neue Methoden wie CBASP, Schematherapie sowie achtsamkeitsbasierte Behandlung. Das anschliessende Kapitel über Stolpersteine und Fallgruben in der Depressionstherapie liefert nützliche Empfehlungen im Umgang mit der Übertragungs- und Interaktionsdynamik depressiver Menschen.

Daniel Hell, langjähriger Klinikdirektor, Ordinarius für Klinische Psychiatrie an der Universität Zürich und heute Leiter des Kompetenzzentrums für Depression und Angst der Klinik Hohenegg, ist ein über die Schweiz hinaus bekannter Wissenschaftler für affektive Erkrankungen. Seine profunde Sachkompetenz kommt in diesem Werk - einmal mehr zum Ausdruck. Eindrucksvoll ist seine engagierte, anteilnehmende und - in guter Bleulerscher Tradition - sich am individuellen Patienten orientierende Grundhaltung. Mit leichter Feder und grosser Offenheit gibt er Einblick in seine eigene Erlebniswelt und therapeutische Arbeitsweise in der Begegnung mit depressiven Menschen. Dies erlaubt dem Leser gewissermassen ein «Lernen am Modell».

Was das Werk zudem auszeichnet, ja einzigartig macht, ist die im gesamten Buch aufscheinende und im Schlusskapitel brillant zusammengefasste depressionsbezogene Auseinandersetzung mit Kultur und Zeitdiagnostik. Hell sieht das Subjekt in der Spätmoderne in besonderer Weise verletzbar und depressionsgefährdet. Er beschreibt prägnant und zutreffend, wie unter den «vorherrschenden gesellschaftlichen Bedingungen - Globalisierung, Dekonstruktion und Deregulierung» ein tragender Grund und somit das seelische Gleichgewicht für viele Menschen verloren zu gehen droht. Die Volkskrankheit Depression wird so Ausdruck eines sich erschöpfenden Selbst und gleichsam zum Spiegel unserer aktuellen Leistungs- und Erfolgsgesellschaft.

Zum Schluss: Werden die mit diesem Buch avisierten Ziele eines personenzentrierten Depressionsgesamtkonzepts erreicht? Hat es sich gelohnt, das Thema Depression neu als Gleichgewichtsstörung weiterzuschreiben? Therapeuten, die sich mit der herausfordernden Behandlung depressiver Patienten unter heutigen Lebensumständen beschäftigen, sind zur Lektüre und Beantwortung dieser Fragen herzlich eingeladen. 